\title{
ASTEROID SURFACE COMPOSITIONS FROM INFRARED SPECTROSCOPIC OBSERVATIONS: RESULTS AND PROSPECTS
}

\author{
H. P. LARSON
}

\begin{abstract}
Advances in IR detector technology, the increased availability of large aperture telescopes, and the techniques of Fourier transform spectroscopy (FTS) now permit IR $(\lambda>1 \mu)$ spectroscopic observations of asteroid surfaces. Asteroids already observed include Ceres, Vesta, and Eros. These initial results demonstrate that such studies can contribute new data concerning asteroid compositions. Some of the most diagnostic features of mineral spectra are in the IR spectral region, and for featureless spectra characterized only $3 y$ slopes the extension of the spectral reflectivity curve into the IR provides tighter constraints on possible mineralogies. A systematic study of additional asteroids should exploit even further this new observational link to problems of meteorite origin and solar system evolution.
\end{abstract}

The methods of Fourier transform spectroscopy (FTS) which revolutionized the study of planetary and stellar atmospheres at infrared wavelengths a decade ago. are now being applied to solid surface constituents of small objects in the solar system. Unique identifications of ices and rock-forming minerals on the surfaces of asteroids and satellites have already been made with FTS techniques. A detailed review of this activity has been prepared by Larson and Fink (1976a). These results are presently included among the most detailed observational constraints concerning the chemical compositions of asteroidal-sized bodies.

\section{EXPERIMENTAL METHODS}

Since the Fourier method has been effectively exploited in astronomical applications by only a relatively few groups, the technique has acquired a certain mystique that has obscured its basic simplicity. The successful application of these complex-looking instruments to studies of the surfaces of faint solar system objects such as the asteroids and satellites is a consequence of this simplicity, as well as the high efficiency and essentially unlimited spectral resolution of FTS methods. The Fourier spectrometers themselves are adequately described elsewhere, for example, Connes and Michel (1975) and Larson and Fink (1975a). In this paper the FTS method is compared in very general terms with those two other experimental techniques, broad-band filter photometry and narrowband filter spectrophotometry, that are more commonly associated with spectral observations of asteroids. 


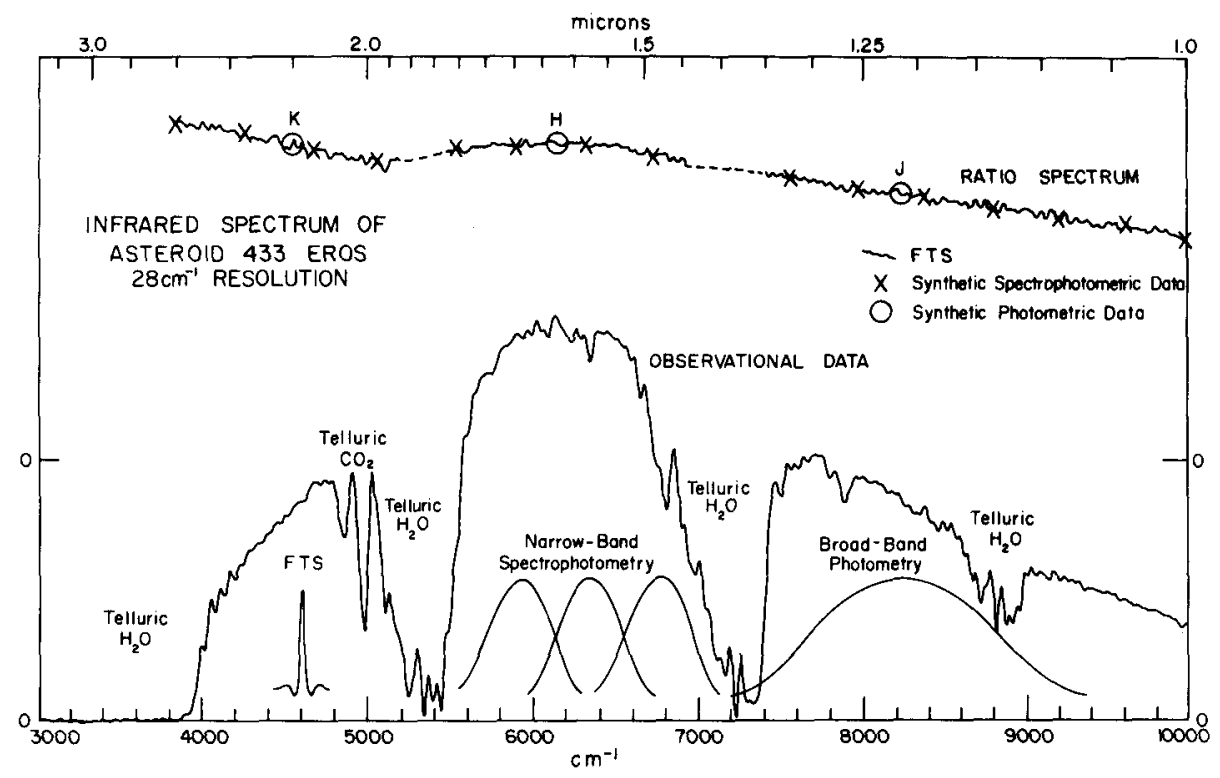

Figure 1. Illustration of the type of IR spectral reflectivity curves produced by three experimental methods: high resolution FTS, narrow-band filter spectrophotometry, and broad-band photometry. The instrumental Iine shapes appropriate to each method are sketched in at the bottom. The synthetic data produced by the two photometric methods are superimposed upon the real FTS data from Larson et al. (1976).

The remote identification of materials on an extraterrestrial object, whether they be gaseous constituents of an atmosphere or solid mineral phases in a rock, depends primarily upon agreement in position between spectral features in the observational data and in some kind of comparison data. Thus, any experiment attempting remote mineralogical analysis must resolve the individual spectral line profiles that are the most reliable indications of the presence of specific materials. The spectroscopic observations of asteroid 433 Eros (Larson et al. 1976) are used to illustrate the ability of each of the three experimental methods under consideration to meet this requirement. Figure 1 contains this data and a schematic representation of the instrumental line shapes (ILS) for the FTS and photometric instruments. The widths of the instrumental line shapes illustrate the fundamental point that each method differs essentially in the spectral resolution that can be achieved with it. Since information content is proportional to spectral resolution, it follows that higher spectral resolution offers greater potential for remote mineralogical analysis. The high resolution FTS data in Figure 1 contain more than 200 spectral elements in the $1-2.5 \mu$ region, all recorded simultaneously (multiplexed). In the spectral reflectivity curve of Eros derived from these observations (top curve, Figure 1), a broad, shallow absorption near $2 \mu$ is superimposed upon a rather red (i.e., increasing reflectivity with wavelength) slope. Larson et al. associated the absorption near $2 \mu$ with pyroxene mineral and the reddened slope with metallic iron. Since the ILS was much narrower than the width of the single absorption feature that was found, no ambiguity surrounded this interpretation.

Spectrophotometric observations of Eros could have been conducted by scanning its spectrum sequentially with a set of narrow-band filters, some of which are sketched in the 1.64 region of Figure 1 . Since no data of this kind actually 
exist for Eros, some were synthesized from the spectroscopic observations by numerical integration with an idealized filter set. The results of these calculations are superimposed upon the spectral reflectivity curve in Figure 1. Although the spectral resolution is reduced by more than an order of magnitude compared to the FTS data, the reddened slope and $2 \mu$ absorption are still evident. It is not certain, however, that real spectrophotometric data would have given such good agreement. Noise in the observations themselves and cumulative error in the data processing procedures could affect the results. It is obvious that the spectrophotometric method will provide less diagnostic data for mineral absorptions narrower than the very broad pyroxene band.

Finally, the results of hroad-band photometric observations of Eros are included in Figure 1. Although real photometric data exist for Eros (Chapman and Morrison 1976) the points in Figure I were synthesized from the spectroscopic data. The three points contained in the $1-2.5 \mu$ region correspond to the $J, H$, and $K$ bands of a standard IR photometer. At this resolution it is no longer possible to identify the $2 \mu$ absorption. The ILS for this photometer is simply too broad to reveal unambiguously even this very broad absorption. These photometric data are consistent with several interpretations, including that actually revealed by higher resolution methods.

Only FTS techniques provide sufficient spectral revolution to resolve the natural line profiles of all features anticipated in mineral spectra. The resolution achieved in specific observations is then dictated by factors independent of the design of the instrumentation itself, one requirement of an optimized experiment. Note that the ability to make absolute flux measurements, which is central to most photometric systems, is not important for spectral analysis hased primarily upon relative response. Although an FTS could produce absolute spectral reflectivities, avoiding this requirement eliminates steps in the data reduction procedures that could lead to appreciable systematic error. It is also probable that the multiplexed operation of an FTS is superior to sequential flux measurements in minimizing the effects at infrared wavelengths of variable transmission of the earth's atmosphere. The $1-2.5 \mu$ region is divided into three major atmospheric transmission windows by strong telluric $\mathrm{H}_{2} \mathrm{O}$ vapor absorptions (see Figure 1). The atmospheric water vapor content is notorious 1 y unpredictable and its effect upon photometric observations can be devastating if not very carefully monitored with IR extinction measurements. In the FTS method all spectral elements simultaneously experience variations in the transmission of the earth's atmosphere, greatly reducing the possibility of systematic error. Also, since no extinction corrections are required for relative flux measurements, much less demand is made upon observations of comparison objects required for data reduction procedures.

\section{IR OBSERVATIONS OF ASTEROIDS}

The following asteroids have been observed spectroscopically in the nearIR (1-2.5 ) : Vesta (Larson and Fink 1975b), Eros (Larson et al. 1976), and Ceres (larson and Fink 1976b). To this list can be added similar observations of the satellites of Jupiter (Pilcher et al. 1972; Fink et al. 1973) and Saturn (Fink et a1. 1976). The recent results for Saturn's satellites (Rhea, Dione, Tethys, and Iapetus) actually define the present lower limit to brightness that can be observed, and which equally applies to ohservations of asteroids. Spectral reflectivity curves at a resolution of about $50 \mathrm{~cm}^{-1}$ can now be produced for any asteroid brighter than about 11 th magnitude (visual) observed through a large aperture telescope ( $2.5 \mathrm{~m}$ or larger) with up to several nights' integration time ( $6 \mathrm{hrs}$. maximum per night). This includes all numbered asteroids between $1-23$ as well as several dozen others. Of course, the ability to make these measurements does not in itself justify expending such large amounts of telescope time 
on them. The spectroscopic method will continue to be reserved for only those objects promising more than routine spectral behavior.

\section{INTERPRETATION OF IR SPECTRAL REFLECTIVITIES OF ASTEROIDS}

The successful acquisition of observational data for asteroid surfaces does not by itself always lead to immediate scientific results. Supporting laboratory spectral studies of minerals are a very necessary accompaniment to an asteroid observing program. Fortunately, much useful diagnostic material already exists. Laboratory comparison spectra of individual rock-forming minerals are readily available (Hunt, Salisbury, and Lenhoff 1974; Adams 1975). For the pyroxene group in particular, empirical calibrations of the systematic shifts of absorption band positions with chemical composition permit detailed analyses of these minerals when observed on remote objects (Adams 1974). Knowledge of the properties of mixtures of these basic rock-forming minerals is especially important for the interpretation of observational data. One approach is through controlled lahoratory experiments that demonstrate the effects of certain spectroscopically active minerals when mixed with other phases (Johnson and Fanale 1973; Adams 1974; Nash and Conel 1974). A second important approach is through laboratory spectral studies of meteorites (Gaffey 1974,1976; Salisbury, Hunt, and Lenhoff 1975). These extraterrestrial samples represent particular mixtures of minerals that presumably formed in or near the present asteroid belt, although cometary origins for some types may be possible. The correlation of the known chemical compositions of these meteorites with their spectral reflectivity characteristics provides diagnostic material that can be directly applied to the interpretation of asteroid spectral data. A few of these comparison spectra of minerals and meteorites are presented below, but the individual papers must be consulted for detailed discussion of all features.

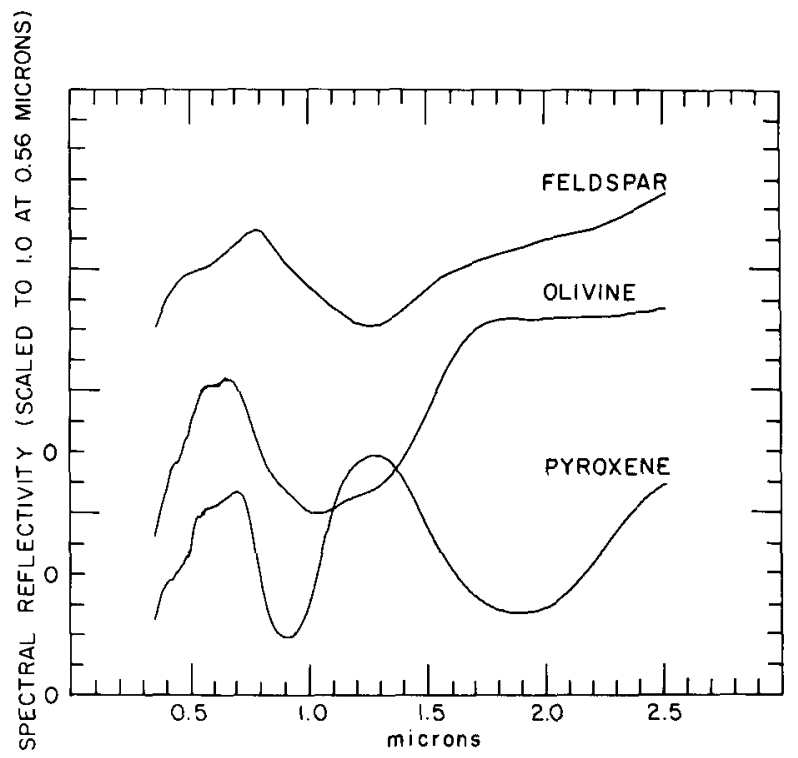

Figure 2. Representative spectra of the three basic rock-forming silicate mineral groups. Ferrous iron (Fe ${ }^{2+}$ ) is responsible for all of the prominent $I R$ absorptions. For iron-deficient silicates, these characteristic absorptions weaken or even disappear. The broad $2 \mu$ absorption is unique to the pyroxenes. Figure adapted from Gaffey (1976). 
Most of the prominent absorption features observed in the near-IR $(1-2.5 \mu)$ in common rock-forming minerals are produced by ferrous iron ( $\mathrm{Fe}^{2+}$ ), hydrated $\mathrm{H}_{2} \mathrm{O}$, and the hydroxyl ion $\left(\mathrm{OH}^{-}\right.$) (Hunt and Salisbury 1970). The positions and appearances of these absorptions are influenced by the type of site occupied and the crystal fields experienced by these ions in particular mineral lattices. To illustrate this point Figure 2 compares the spectral reflectivities of three important rock-forming mineral groups, the pyroxenes, olivines and feldspars. Although the broad absorptions in each spectrum are all produced by $\mathrm{Fe}^{2+}$, the

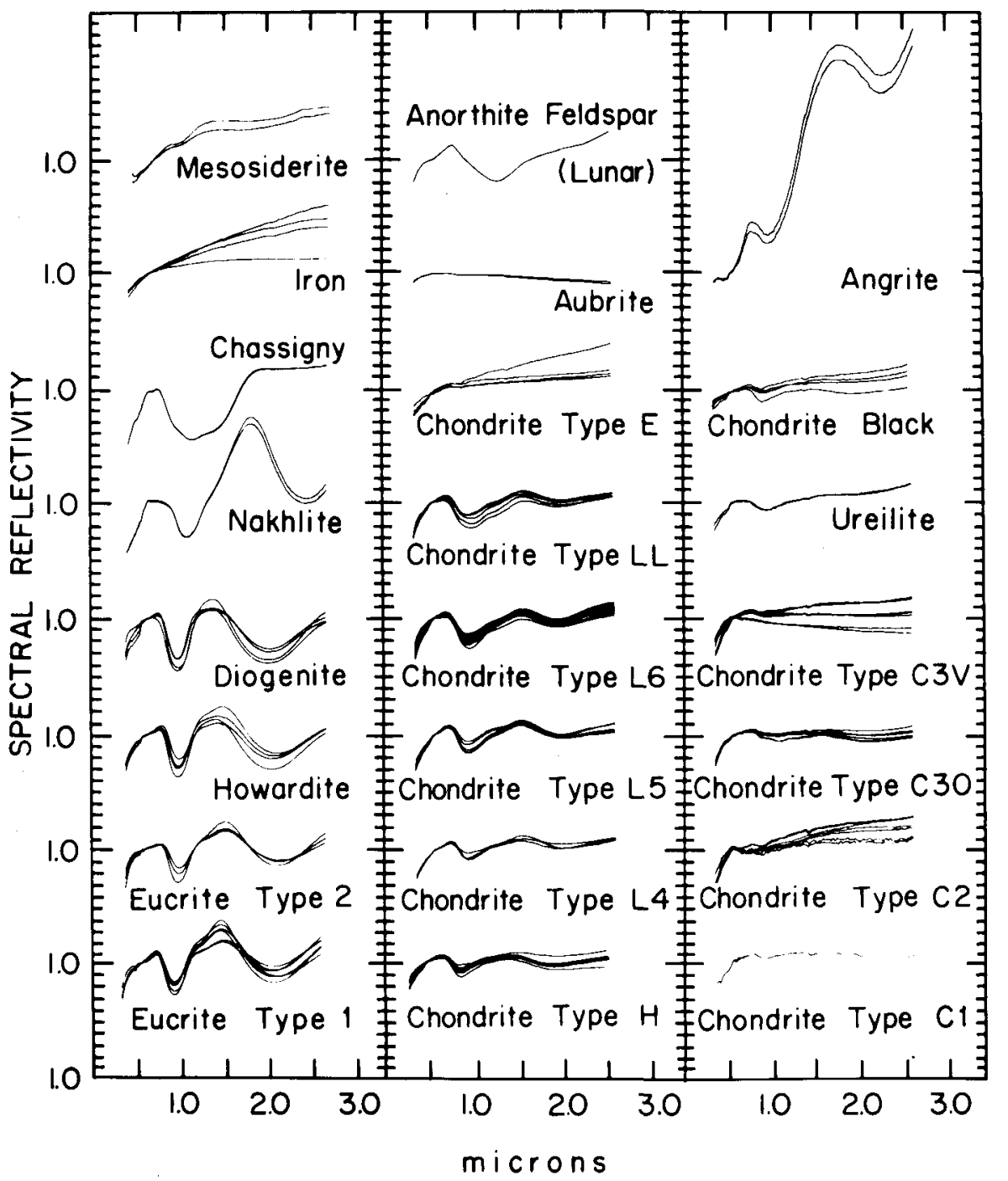

Figure 3. Spectral reflectivity curves for the meteorite classes. Pyroxene bands produce the more obvious spectral features. Even among the featureless spectra, there are important differences in slope that can sometimes be exploited. Figure courtesy of M. J. Gaffey. 
bands appear sufficiently different that each mineral can be uniquely identified. Moreover, only very low resolving power $(\lambda / \Delta \lambda \sim 100)$ is required to reveal these bands, a definite advantage when flux is limited. Although both the olivines and pyroxenes possess absorptions neàr $1 \mu$, which can lead to some confusion in interpreting astronomical data, only the pyroxenes display the prominent $2 \mu$ band whose presence or absence in IR data then becomes a definitive test for this mineral.

Figure 3 contains Gaffey's spectral reflectivity curves for the meteorite classes. Although these spectra constitute a rather complete set of comparison data of known meteorite types, they probably do not represent all mineralogical assemblages that existed in the meteorite parent bodies. A few meteorites are nearly pure mineral phases, such as Chassigny which is about $95 \%$ olivine, but most are mixtures of the three minerals in Figure 2 along with metallic $\mathrm{Ni}-\mathrm{Fe}$, hydrated and hydroxylated silicates, iron oxides, and numerous other minor constituents. The orthopyroxene bands, for example, are very obvious in the spectra of the basaltic achondrites (eucrites, howardites, etc.) and, though weaker, in the type $\mathrm{H}$ and $\mathrm{L}$ ordinary chondrites, The weaker feldspar absorption at about $1.25 \mu$ can be seen in the type 1 eucrites and in the type L chondrites, but it nearly disappears in a howardite where feldspar constitutes less than about a third of the bulk composition. The carbonaceous chondrites would have displayed rather distinctive spectra were it not for the presence of small amounts $(<10 \%)$ of opaque minerals (iron oxides, carbonaceous matrix material) that completely suppress the prominent absorptions of these meteorites' major minerals. The absence of ferrous iron bands in the type $E$ chondrites illustrates the bland spectra produced by iron-free silicates, and the reddened reflectivity of iron meteorites is characteristic of metallic $\mathrm{Ni}-\mathrm{Fe}$.

The interpretation of asteroid spectra usually starts by comparing them with meteorite spectra. The results of this screening establish what, if any, detailed compositional inferences can be made. Using asteroid Eros again as an example, Larson et al. (1976) could not match its spectral reflectivity (see Figure 1) with any of the meteorite spectra in Figure 3 . They did note, however, that its spectral reflectivity might be more closely approximated hy combining the reddened reflectivity of an iron meteorite with the highly equilibrated silicates found in basaltic achondrites. No other IR spectroscopic data exist for Eros, but as wavelengths below $1 \mu$ several experiments were conducted with spectrophotometric (Veeder et al. 1976; Pieters et al. 1976) and higher resolution spectroscopic (Wisniewski 1976) techniques. Analyses of these spectral 'reflectivity curves below $1 \mu$ differed as to the nature of the silicate component on Eros, but all identified metallic Ni-Fe as an important constituent. This implies that Eros is a "stony-iron" assemblage not represented among known meteorite types, although the differences may not be great and may be explained as well by factors other than composition alone. The "Eros issue" of Icarus (Volume 28, Number 1, 1976) should be consulted for further details regarding the composition of this asteroid.

Other asteroids, including the largest, Ceres, do not closely match known meteorite types either (Chapman and Salisbury 1973). For Ceres in particular, its low albedo and featureless spectrum suggest carbonaceous chondrite material. Johnson and Fanale (1973) demonstrated through laboratory experiments that simple adjustments of the mineralogical constituents of carbonaceous chondrite material, particularly the opaque components, could closely reproduce Ceres' observed spectral reflectivity, at least out to about $1 \mu$. The uniqueness of these mortar and pestle "solutions" can be further tested by fitting as much of the asteroid's observed spectral reflectivity as possible. The addition of the IR portion of this curve more than triples the spectral range available, which for Ceres and similar, featureless spectra may be quite influential in limiting possible mineralogies. Preliminary analysis of IR observations of Ceres (Larson and Fink 1976b) indicates, in fact, that Ceres exhibits spectral characteristics 
that, among carbonaceous chondrite meteorites, are developed only in type $C 2$ material.

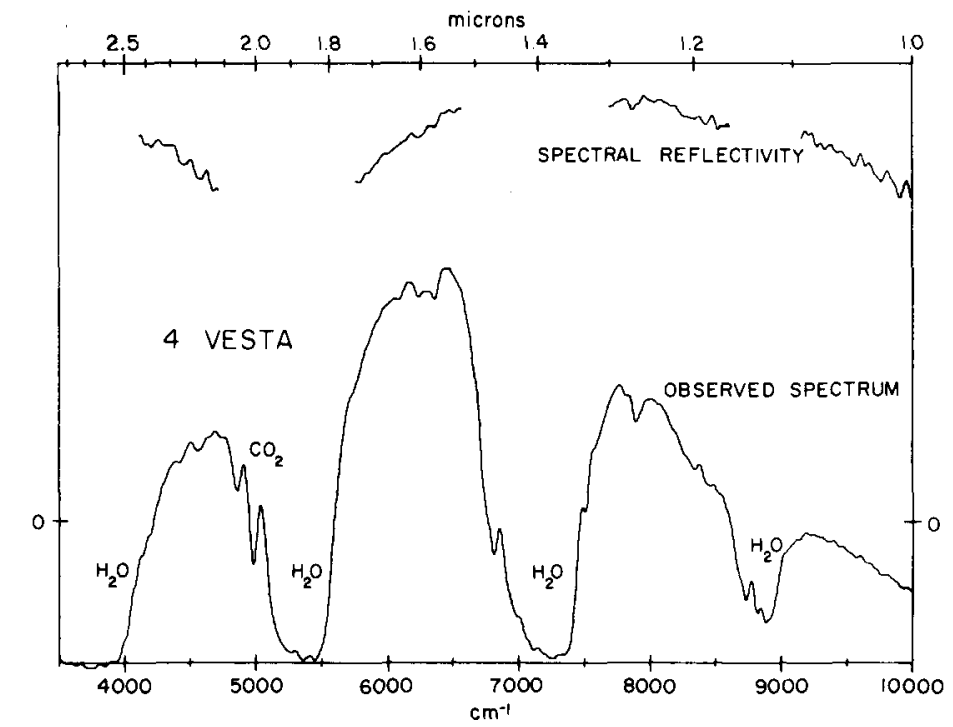

Figure 4. FTS observations of Vesta $\left(R-50 \mathrm{~cm}^{-1}\right)$ during its 1975 apparition. The pyroxene band near $2 \mathrm{H}$ is very obvious even though strong atmospheric water vapor absorptions have obscured about half the spectral reflectivity curve.

In contrast to the interpretations outlined above, spectral observations of asteroid Vesta have been readily associated with basaltic achondrite meteorite types as a consequence of its deep pyroxene bands (McCord et al. 1970; Larson and Fink 1975b). Subsequent observations of this asteroid have strengthened this interpretation. Figure 4 contains the most recent FTS data recorded at the Steward Observatory 2.3m telescope in August 1975. The deep, broad pyroxene band at $2 \mu$ dominates Vesta's IR spectral reflectivity (compared with the pure pyroxene spectrum in Figure 2). The position of this band can be related to the detailed chemical composition of Vesta's pyroxene (Adams 1974). Unfortunately, very moist atmospheric conditions at the time of these observations resulted in Vesta's $2 \mu$ pyroxene band being heavily obscured by the $1.9 \mu$ telluric $\mathrm{H}_{2} \mathrm{O}$ vapor band. The pyroxene absorption maximum cannot be located with the precision that would have been possible under drier atmospheric conditions. The absorption maximum is estimated to be at $2.0 \mu$ with a rather large uncertainty of $\pm 0.05 \mu$, after compensating for the non-solar colors of the comparison object. This IR position can be converted to a specific pyroxene using the pyroxene band-band plot in Figure 5 devised by Adams. The most recent determination of the com-

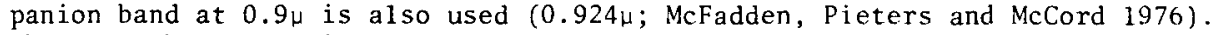
These two band positions place Vesta exactly on the pyroxene line in Figure 5 in the region occupied by high-iron orthopyroxenes (hypersthenes) and low-calcium clinopyroxenes (pigeonites) or, in terms of meteorite types, eucrites.

\section{IMPLICATIONS AND CONCLUSIONS}

Determining the compositions of asteroidal-sized objects is not only an 


\section{LARSON}

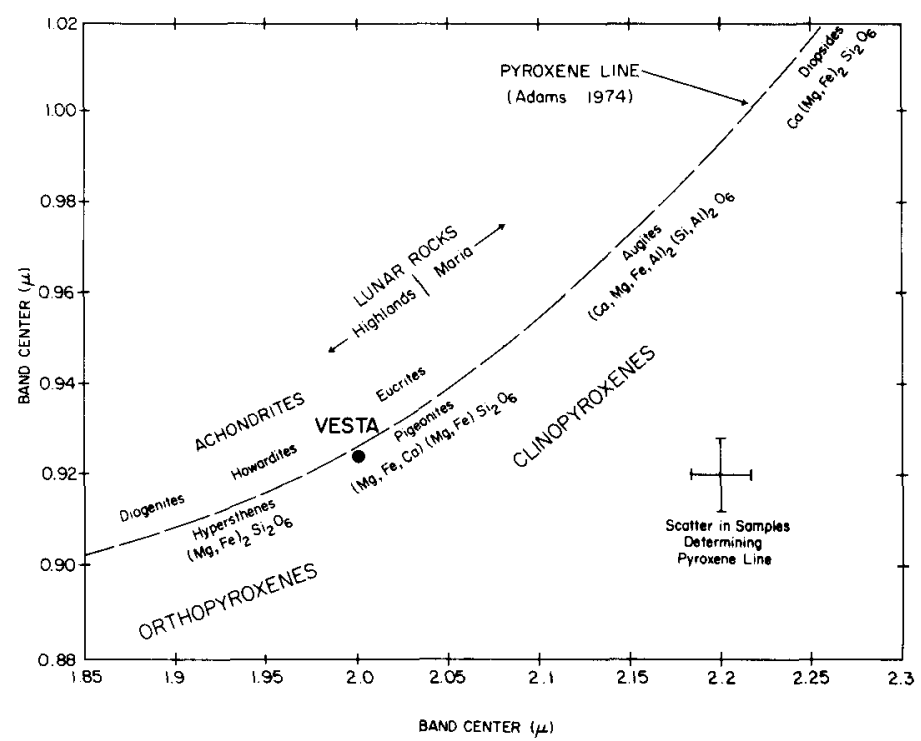

Figure 5. Pyroxene band-band plot. Both the 0.9 and 2.04 bands of pure pyroxenes shift systematically with mineral type, defining a "pyroxene line" useful for interpretation of remotely observed pyroxenes. The most recent analyses of Vesta's puroxene bands locate this asteroid on the pyroxene line which strengthens the case for igneous differentiation on this object. Figure revised from Larson and Fink $(1975 b)$.

important end in itself, but these results also contribute to broader questions of solar system origin and evolution. Studies of the nature and distribution of carbonaceous chondrite material on asteroids such as Ceres may provide a vital observational link to the origin of organic matter in extraterrestrial environments. Vesta's close association with basaltic achondrite meteorites suggests igneous differentiation in asteroidal-sized bodies. In this case remote mineralogical sensing has provided in situ evidence for a heating episode early in the thermal history of the solar system. The unrecognized stony-iron mixture on Eros may eventually be better understood through laboratory studies. The spectral characteristics of known types of stony-iron meteorites are poorly determined at present, and the origin of the metallic $\mathrm{Ni}-\mathrm{Fe}$ in some of them, mesosiderites in particular, is baffling in spite of detailed laboratory analyses.

The remote identification of solids on asteroid surfaces has emerged as a distinct area of planetary astronomy only since about 1970. The FTS measurements are even more recent contributions to this field that can best grow by combining all types of observational and diagnostic material. The FTS techniques have benefited from these initial efforts as evidenced by the steady improvement in SNR in subsequent observations of Vesta and by the successful observations of much fainter objects. As higher quality IR data accumulate on a growing list of asteroids, increased attendion must be devoted to more sophisticated analyses that extract all of the information contained in such data. Thus, laboratory simulations and computer syntheses are among the analytic tools that will increasingly replace simple visual comparison of data sets.

This research acknowledges support from NASA Grant NSG 7070. 


\section{REFERENCES}

Adams, J. B. 1974, J. Geophys. Res., 79, 4829.

Adams, J. B. 1975, in Infrared and Raman Spectroscopy of Lunar and Terrestrial Minerals, (New York: Academic Press, 1975), p. 9].

Chapman, C. R., and Sal i sbury, J. W. 1973, Icarus, 19, 507.

Chapman, C. R., and Morrison, D. 1976, Icarus, 28, 91.

Connes, P., and Michel, G. 1975, Appl. Opt. 14, 2067.

Fink, U., Dekkers, N. H., and Larson, H. P. 1973, Astrophys. J. Lett., $179,1087$.

Fink, U., Larson, H. P., Treffers, R. R., and Gautier, T. N. 1976, Astrophys. J. Lett., 207, L63.

Gaffey, M. J. 1974, Ph.D. Thesis, Mass. Inst. Tech., Cambridge.

Gaffey, M. J. 1976, J. Geophys. Res., 81, 905.

Hunt, G. R., and Sali sbury, J.W. 1970, Modern Geologe, 1, 283.

Hunt, G. R., Salisbury, J. W., and Lenhoff, C. J. 1974, Modern Geology, 5, 15., contains references to previous papers in series.

Johnson, T. V., and Fanale, F. P. 1973, J. Geophys. Res., 78, 8507.

Larson, H. P., and Fink, U. 1975a, Appl. Opt., 14, 2085.

Larson, H. P., and Fink, U, 1975b, Icarus, 26, 420.

Larson, H. P., Fink, U., Treffers, R. R., and Gautier, T. N. 1976, Icarus, 28, 95.

Larson, H. P., and Fink, U. 1976a, submitted to Appl. Spectrosc.

Larson, H. P., and Fink, U. 1976b, in preparation.

McCord, T. B., Adams, J. B., and Johnson, T. V. 1970, Science, 168, 1445.

McFadden, L., Pieters, C., and McCord, T. B. 1976, Bull. Am. Astron. SoC., in press.

Nash, D. B., and Conel, J. E. 1974, J. Geophys. Res., 79, 1615.

Pieters, C., Gaffey, M. J., Chapman, C. R., and McCord, T. B. 1976, Icarus, 28, 105 .

Pilcher, C. B., Ridgway, S. T., and McCord, T. B. 1972, Science, 178, 1087.

Sal isbury, J. W., Hunt, G. R., and Lenhoff, C. J. 1975, Modern Geology, $5,115$.

Veeder, G. J., Matson, D. L., Bergstrahl, J. T., and Johnson, T. V. 1976, Icarus, 28, 79 .

Wisniewski, W. 2 . 1976, Icarus $28,87$.

\section{DISCUSSION}

JOHNSON: These are beautiful spectra you are showing us. I'd like to point out that we published broad band infrared data at 1.6 and $2.2 \mathrm{\mu m}$ of Ceres a couple of years ago (Johnson et a1., Ap.J., 197, 527, 1975). We compared these data with laboratory data (Johnson and Fanale, J. Geophys. Res., 89, 8507, 1973) and also came to the conclusion that Ceres is covered by material similar to $C_{2}$ carbonaceous chondrites. This also illustrates the use of the broad band data for surveys when dealing with relatively featureless spectra.

WHIPPLE: I believe that space erosion from particles should affect the surface characteristics of asteroids. Because space erosion decreases with solar distance it will therefore produce a systematic effect. If sputtering darkens material to saturation, less erosion should shorten the time scale at greater solar distances. Hence darker asteroids should occur at greater solar distances, as observed.

BRECHER: Would you be able to resolve by this sensitive IR technique between spectra of bound water (e.g., hydrated silicates), water vapor and ice? If so, it would be important for both asteroid surfaces and comets. Did you see it on ceres? 
LARSON: Water as vapor, solid, hydrate or hydroxyl (OH-) can readily be distinguished on the basis of near-IR spectra. In observational data, unfortunately, water vapor in the earth's atmosphere masks the diagnostic spectral features of extraterrestrial water. Only the spectral features of water ice remain obvious. Trace amounts of water vapor on extraterrestrial objects can best be detected from special high altitude sites such as aircraft. Hydrated and hydroxylated water may be detectable in ground-based observations under conditions of very low atmospheric water vapor. Our Ceres data were recorded under such conditions, but no evidence is seen for bound water on this asteroid.

DICKEL: What is present at 3-4000 $\AA$ ? All curves fall off there.

ZELLNER: The ultraviolet drop off is usually attributed to the long-wavelength wings of strong $U V$ absorptions due to transition-metal ions, such as $F^{2+}$, in a silicate lattice.

GOLD: Solar wind sputtering must have occurred on these bodies, and indeed proceeded almost certainly to saturation of the optical effects. On most terrestrial rocks this has profound effects, almost always causing intense darkening. $I$ do not know the infrared effects, but $I$ would consider it quite unsafe to make any deductions from a comparison of laboratory meteoritic spectra with these bodies, without having first assured saturation simulated solar wind bombardment of the samples.

GAFFEY: With regard to Gold's suggestion of asteroid spectral modification by sputtering, we have considered this problem by analogy to the modification of optical properties of the lunar rocks to soils and breccias. The spectral behavior of the majority of asteroids either in overall shape or in absorption band intensity is inconsistent with any significant mineralogical, petrological or chemical modification of the type exhibited by the lunar samples.

JOHNSON: I'd like to support Mike Gaffey's point. The variability of asteroid spectra observed indicates that they have not been reduced to uniform color by space weathering or sputtering. In fact one of the conclusions which rom McCord, John Adams, and I drew about Vesta's spectrum, particularly its deep absorption band, was that there has not been alteration of the optical properties as there has been on the lunar surface. We now have evidence that other asteroids have also apparently not been so weathered. I think this is very interesting in terms of what processes operate in different parts of the solar system.

GROSSMAN: What mineralogical features of $\mathrm{C} 2$ chondrites make their spectra different from those of $\mathrm{Cl}$ chondrites?

LARSON : Two problems prevent a definitive answer to this question:

a) The spectral reflectance characteristics of $C l$ meteorites are poorly established since only one sample is available for study (Gaffey 1974,1976).

b) The spectroscopically active component of C2 material may not be the opaque component (magnetite, carbonaceous matrix) since Gaffey's studies suggest a role for the silicate component (montmorilionite, etc.).

Not much can be done about the first problem, but current laboratory studies involving the second may soon provide at least a partial answer to your question. 